\title{
An unusual case of spontaneous bilateral basal ganglia bleed
}

\author{
Jessica Kayastha ${ }^{1}$, Pravesh Rajbhandari ${ }^{1}$, Pritam Gurung ${ }^{1}$, Bishal Shrestha ${ }^{1}$, Sambardhan \\ Dabadi $^{1}$, and Basant Pant ${ }^{2}$ \\ ${ }^{1}$ Annapurna Neurological Institute and Allied Sciences \\ ${ }^{2}$ Annapurna Neurological Institute and allied Sciences
}

October 20, 2021

\begin{abstract}
Spontaneous bilateral intracerebral hemorrhage is an outstandingly infrequent context. Ensuing hypertension, trauma inflicted to the brain case is another common cause that out turn into the sequelae of bilateral intracerebral hemorrhage. Lately, a few cases of bilateral basal ganglia bleed have been revealed, as a repercussion of COVID-19 infection.
\end{abstract}

\section{INTRODUCTION}

In the third world countries, the leading cause of basal ganglia bleed has been ascribed to hypertension. Amidst these patients, the mode of presentation is hemiplegia or features provocative of raised intracranial pressure. Most of them have either been diagnosed with hypertension not under medication or those who have refrained to anti-hypertensive medications prescribed.

Venous sinus thrombosis, hemorrhagic diathesis, arteriovenous malformation, vasculitis, amyloid angiopathy, aneurysm, neoplasm, drug abuse and angioma has been deemed answerable for multiple intracerebral hemorrhages [1]. Moreover, bilateral basal ganglia bleed has also been accredited to trauma, hyperglycemic hyperosmolar syndrome, diabetic ketoacidosis, methanol poisoning, migraine, anticoagulant use, lighting strike and fungal infection to name a few [2-6].

CT scan of the head demonstrates presence of hyper dense lesion in basal ganglia. DSA or CTA of Circle of Willis are inconclusive. We hereby report a case of simultaneous bilateral ICH with involvement of both the basal ganglia treated conservatively with an up to par outcome.

\section{CASE PRESENTATION}

A 39 -years- male patient presented to our institution with the history of acute onset of weakness of the right side of the body, deviation of angle of the mouth to left side, generalized stiffness of the body, unable to speak and urinary incontinence for which he was evaluated and managed conservatively in another center. His medical, surgical and drug history was unremarkable. Family history was irrelevant and history of trauma was trivial.

On retrospective survey, when he presented to the ER in the other center, his pulse rate was $98 \mathrm{bpm}$, blood pressure was 200/100 mm of $\mathrm{Hg}$, Glasgow coma scale (GCS) was E4 V2 M6, pupils were bilateral equal and reactive to light. Further neurological examination revealed a decreased sensation over right side of the body with motor power in the Right upper and lower limbs was $0 / 5$ and $5 / 5$ in left upper and lower limbs. His deep tendon reflexes were absent on the right side but preserved on the left side. Similarly, the plantar reflex on the right side was extensor and flexor on the left.

Laboratory parameters were within normal limit. CT scan of head revealed hyper dense lesion involving bilateral basal ganglia suggestive of blood with volume of $28 \mathrm{cc}$ in left and $13 \mathrm{cc}$ in right (Figure 1). He was 
then admitted in an ICU and managed conservatively. There was gradual improvement in the condition of patient, he was then shifted to ward and after 9 days of admission, he was discharged and he presented to our center for further management and advice.

At presentation to our center, GCS: E4 V4 M6, Pupils: Bilateral $2 \mathrm{~mm}$ and reactive to light, Sensation was decreased over right half of the body. Motor power in the RUL/RLL was $4+/ 5$ and in LUL/LLL was $5 / 5$, deep tendon reflexes were decreased over right side.

CT head and CTA was repeated. CT head revealed resolving hematoma (Figure 2). CTA was negative for intracranial aneurysms, vascular malformations, vasospasm or cerebral venous thrombosis (Figure 3).

When the patient presented to our facility six months following the first bleed, he was fully oriented to time, location, and person, with motor power $5 / 5$ in all four limbs and very little slurring of speech and enhanced emotional drive. He is now able to carry out his daily tasks alone and has returned to his farm. We took another CT scan of the head, which revealed a resolving hematoma. (Figure: 4).

\section{DISCUSSION}

Contrary to a frequent presentation of unilateral BG bleed, finding a bilateral basal ganglia bleed is out of the ordinary. The aetiopathogenesis in the circumstance of simultaneous bilateral basal ganglia hemorrhage remains unsettled. A handful of hypothesis has been postulated by different authors with reference to aforementioned context. The most common plausible pathogenesis is the concurrent rupture of bilateral micro aneurysms on lenticulostriate arteries by chance [7]. Other mechanism predicate that the initial hemorrhage results in specific hemodynamic ambience, such as reflex increase in blood pressure, evoking rupture of a second micro aneurysm on the contralateral side in a brief time span, which seems more credible[7] . Nonetheless, traumatic basal ganglia bleeding is uncommon, and bilateral basal ganglia hemorrhage after trauma is extremely rare. Concerning the trauma, two hypotheses has been put forward namely spontaneous or traumatic hemorrhage [8]. Spontaneous hemorrhage occurs when the blood pressure haul up impulsively due to emotional or physical strain and ruptures the lenticulostriate artery forming a hematoma. The genesis to this predicament being congenital vascular malformation, long term hypertension and diabetes mellitus [9]. Traumatic hemorrhage hypothesis surmise shearing force that cause the tear in the palladium branch of the anterior choroid artery. When a hefty coercion is imposed to the vertex, forehead, or occipital area directed towards the tentorium, there would be a reposition of the brain parenchyma through the tentorial notch with shredding and ripping of vessels by shearing forces, resulting in hemorrhages in the basal ganglia region. A few authority labels this as intermediary contusions [10]. The basal ganglia and thalami are profoundly metabolic brain domains, making them susceptible to hypoxic-ischemic injury, toxic poisoning (carbon monoxide, methanol, cyanide), metabolic anomalies (liver disease, Leigh disease, Wilson disease, hypoglycemia, and osmotic myelinolysis), and neuro degeneration [11]. The bilateral basal ganglia or thalami can also be targeted by focal flavi virus infection, toxoplasmosis, and primary CNS lymphoma $[11,12]$. Lately, with the emergence of global pandemic, the sinner being the SARS-CoV-2, it has been presumed that the ailment can be neurotropic via the trans cribriform or the hematogenous route [13]. The doubt as to why COVID 19 would predispose to bilateral ganglia hemorrhage remains open and undecided. Even so, the virion has been reported to predispose to a vulnerable hypercoagulable state, increasing the likelihood of cerebrovascular accident and ischemic stroke [14]. It is theorized that the aetiopathology of COVID 19 whether by direct invasion or via systemic inflammatory responses, may contribute to bilateral basal ganglia hemorrhage and to ensuing neurological deficits [15].

As outrageous it is to involve the bilateral basal ganglia, an impoverished outcome is always anticipated. Hereupon, debate come to light whether or not to intervene the patient surgically. On account of destruction of crossing and non-crossing fibers, bilateral diaschisis phenomenon, severely disrupted and altered level of consciousness, quadriparesis and pseudo bulbar palsy, compromised aftermath is contemplated [16]. While some professionals prefer an upfront evacuation of hematoma depending upon the volume, the others favor to lie in wait to undertake surgery immediately when there are signs of surge in intracranial pressure.

As luck would have it, our patient achieved an admissible recovery. We found it compelling that the patient 
could circumvent surgical intervention in the face of voluminous hematoma with bilateral involvement. Into the bargain, his neurological function also rectified making him a self-sufficient being.

\section{CONCLUSION}

Bilateral basal ganglia bleed is an unusual surgical context. The most common predisposing factors are hypertension and trauma. Different hypothesis has been put forward and postulated by gallant authorities, but the exact architecture of pathophysiology leading to its evolution is open to debate.

\section{ABBREVIATION:}

CT: Computed tomography

CTA: Computed tomography Angiography

ICH: Intracranial Hemorrhage

DSA: Digital Subtraction Angiography

SBBGH: Simultaneous Bilateral Basal Ganglia Hemorrhage

COVID-19: Corona virus Disease

\section{FIGURE LEGEND:}

Figure 1: CT head showing bilateral BG bleed immediately after Cerebrovascular Accident

Figure 2:CT head after showing resolving hematoma 1 week of Cerebrovascular Accident

Figure 3:CT Angiogram of Circle of Willis

Figure 4:CT Head plain showing resolving hematoma

\section{AUTHOR CONTRIBUTION:}

Jessica Kayastha: Original draft, Conceptualization, review and editing

Pravesh Rajbhandari: Supervision

Pritam Gurung: Review, editing

Bishal Shrestha: Review, editing

Sambardhan Dabadi: Review, editing

Basant Pant: Supervision

\section{ACKNOWLEDGEMENT:}

The authors express their gratitude to Dr. Pramesh Prasad Shrestha for proofreading the manuscript.

\section{CONFLICT OF INTEREST}

None of the authors have potential conflicts of interest to be disclosed.

\section{ETHICS APPROVAL AND CONSENT TO PARTICIPATE}

Ethical approval of case report is not needed in accordance to the local ethical guideline. Written informed consent was obtained from the patient to include the clinical details.

KEY CLINICAL MESSAGE: Simultaneous Bilateral Basal Ganglia Hemorrhage is an exceptionally rare ailment. This genre of cerebrovascular accident embraces a comprehensive span of morbidity and mortality. In an acute setting, CT scan of head is the most relevant imaging.

\section{References}


[1] S. Takeuchi, Y. Takasato, H. Masaoka, T. Hayakawa, H. Yatsushige, T. Sugawara, Simultaneous multiple hypertensive intracranial hemorrhages, Journal of Clinical Neuroscience. (2011). https://doi.org/10.1016/j.jocn.2011.01.020.

[2] N. Permpalung, W. Cheungpasitporn, D. Chongnarungsin, T.M. Hodgdon, Bilateral putaminal hemorrhages: Serious complication of methanol intoxication, North American Journal of Medical Sciences. (2013). https://doi.org/10.4103/1947-2714.120804.

[3] B. Ertl-Wagner, O. Jansen, S. Schwab, K. Sartor, Bilateral basal ganglion haemorrhage in diabetic ketoacidotic coma: Case report, Neuroradiology. (1999). https://doi.org/10.1007/s002340050822.

[4] A. Raabe, U. Krug, Migraine associated bilateral intracerebral haemorrhages, Clinical Neurology and Neurosurgery. (1999). https://doi.org/10.1016/S0303-8467(99)00024-4.

[5] S.J. Cho, T.K. Won, S.J. Hwang, J.H. Kwon, Bilateral putaminal hemorrhage with cerebral edema in hyperglycemic hyperosmolar syndrome, Yonsei Medical Journal. (2002). https://doi.org/10.3349/ymj.2002.43.4.533.

[6] S. M, T. S, K. A, S. T, F. M, M. R, Spontaneous bilateral intracerebral hemorrhage occurring simultaneously-case report, Neurologia Medico-Chirurgica. 26 (1986) 545-547. https://doi.org/10.2176/NMC.26.545.

[7] J. Zhao, Z. Chen, Z. Wang, Q. Yu, W. Yang, Simultaneous bilateral hypertensive basal ganglia hemorrhage, Neurologia i Neurochirurgia Polska. (2016). https://doi.org/10.1016/j.pjnns.2016.03.003.

[8] J.W. Finnie, Forensic Pathology of Traumatic Brain Injury:, Http://Dxdoiorg/101177/0300985815612155. 53 (2015) 962-978. https://doi.org/10.1177/0300985815612155.

[9] Y.X. Zhang, S.Q. Wei, Y.Y. Xing, Q. Liu, W.J. He, Bilateral traumatic hemorrhage of the basal ganglia, Chinese Journal of Traumatology - English Edition. (2016). https://doi.org/10.1016/j.cjtee.2015.11.022.

[10] Gennarelli, Graham, Neuropathology of the Head Injuries., Seminars in Clinical Neuropsychiatry. (1998).

[11] A.N. Hegde, S. Mohan, N. Lath, C.C.T. Lim, Differential diagnosis for bilateral abnormalities of the basal ganglia and thalamus, Radiographics. (2011). https://doi.org/10.1148/rg.311105041.

[12] Y.-X. Zhang, S.-Q. Wei, Y.-Y. Xing, Q. Liu, W.-J. He, Bilateral traumatic hemorrhage of the basal ganglia, Chinese Journal of Traumatology. 19 (2016) 247. https://doi.org/10.1016/J.CJTEE.2015.11.022.

[13] A.M. Baig, A. Khaleeq, U. Ali, H. Syeda, Evidence of the COVID-19 Virus Targeting the CNS: Tissue Distribution, Host-Virus Interaction, and Proposed Neurotropic Mechanisms, ACS Chemical Neuroscience. (2020). https://doi.org/10.1021/acschemneuro.0c00122.

[14] M.F. Goldberg, M.F. Goldberg, R. Cerejo, A.H. Tayal, Cerebrovascular Disease in COVID-19, American Journal of Neuroradiology. (2020). https://doi.org/10.3174/AJNR.A6588.

[15] R. Daci, M. Kennelly, A. Ferris, M.U. Azeem, M.D. Johnson, F. Hamzei-Sichani, A.H. Jun-O'Connell, S.K. Natarajan, Bilateral basal ganglia hemorrhage in a patient with confirmed COVID-19, American Journal of Neuroradiology. (2020). https://doi.org/10.3174/ajnr.A6712.

[16] C.P. Yen, C.L. Lin, A.L. Kwan, A.S. Lieu, S.L. Hwang, C.N. Lin, S.L. Howng, Simultaneous multiple hypertensive intracerebral haemorrhages, Acta Neurochirurgica. (2005). https://doi.org/10.1007/s00701004-0433-y. 


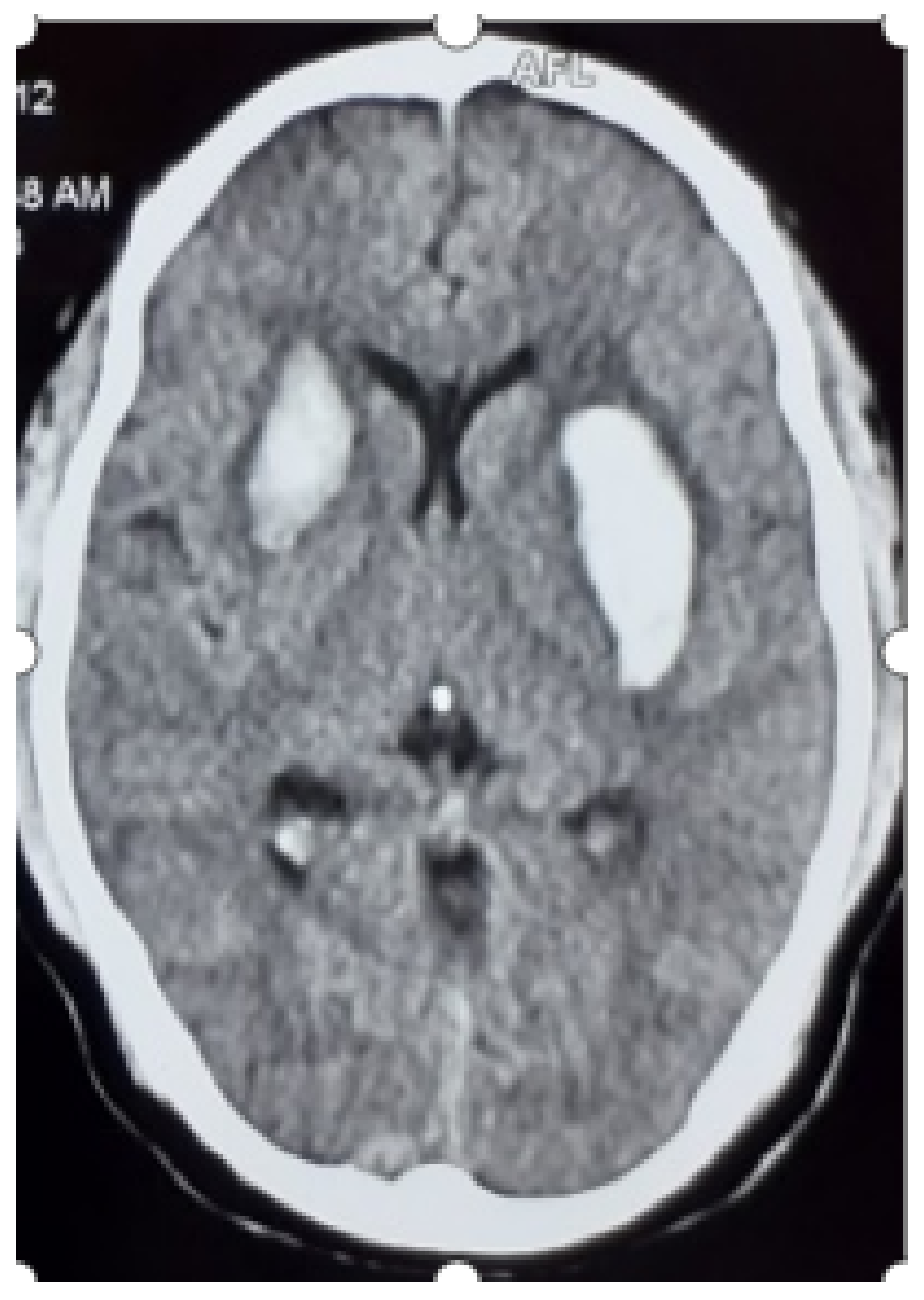









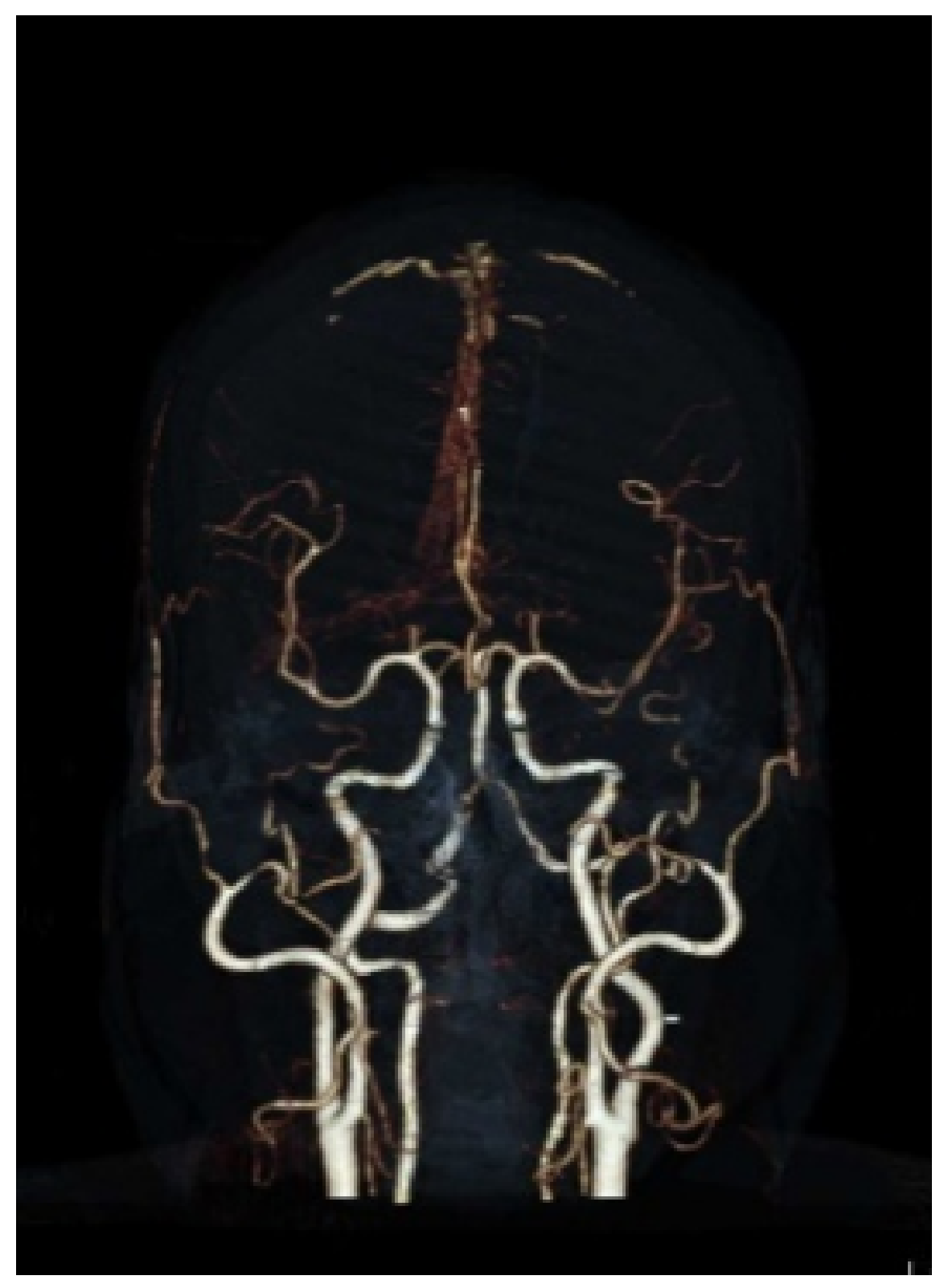




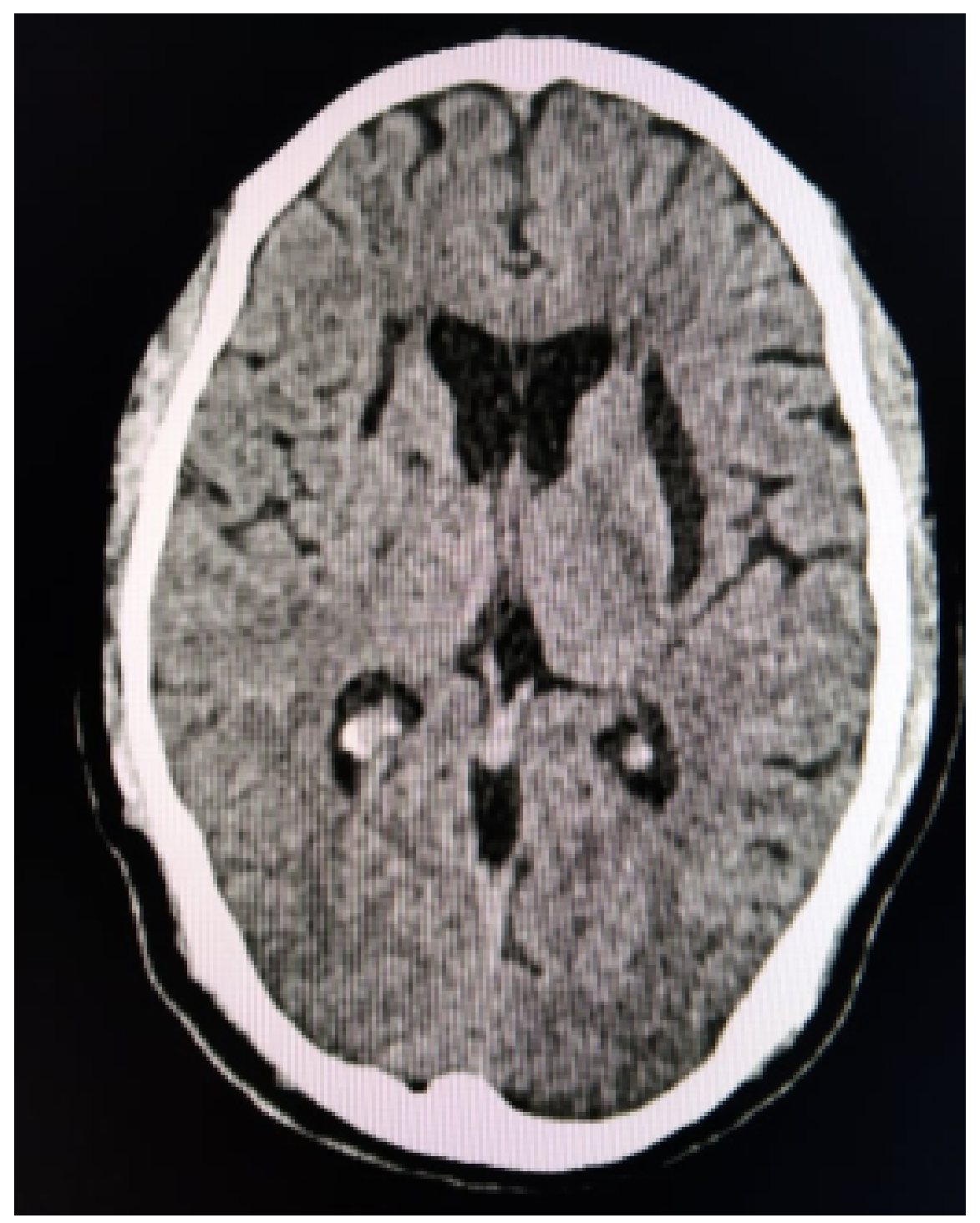

